\title{
Refractory cachexia is associated with increased plasma concentrations of fentanyl in cancer patients
}

\author{
Manabu Sunol,* \\ Yuriko Endo ${ }^{1, *}$ \\ Hiroyuki Nishie ${ }^{2}$ \\ Makoto Kajizono ${ }^{3}$ \\ Toshiaki Sendo 3 \\ Junji Matsuoka ${ }^{4}$ \\ 'Department of Oncology \\ Pharmaceutical Care and Science, \\ Graduate School of Medicine, \\ Dentistry and Pharmaceutical \\ Sciences, ${ }^{2}$ Department of \\ Anesthesiology and Resuscitology, \\ Graduate School of Medicine, \\ Dentistry and Pharmaceutical \\ Sciences, Okayama University, \\ ${ }^{3}$ Department of Pharmacy, Okayama \\ University Hospital, ${ }^{4}$ Faculty of Health \\ Sciences, Okayama University Medical \\ School, Okayama, Japan \\ *These authors contributed equally \\ to this work
}

This article was published in the following Dove Press journal:

Therapeutics and Clinical Risk Management

8 May 2015

Number of times this article has been viewed

Background: An appropriate plasma concentration of fentanyl is the key to achieving good pain control in cancer patients. Cachexia, a multifactorial syndrome, is known to affect drugmetabolizing enzymes. However, the fentanyl concentrations in the blood of patients with cachexia have not been analyzed. The aim of this study was to evaluate the influence of cancer cachexia on dose-adjusted plasma fentanyl concentrations in cancer patients.

Methods: Blood was collected from 21 Japanese cancer patients treated with a 24-hour transdermal fentanyl patch during the steady state of fentanyl plasma concentration. Plasma fentanyl concentrations were analyzed using liquid chromatography with tandem mass spectrometry (LC-MS/MS), and the levels were adjusted with the dose of fentanyl. Laboratory data were collected, and the cachexia stage was determined, based on study by Fearon et al. Multiple regression analysis was performed to identify the factors that affected fentanyl plasma concentrations.

Results: Eight patients were classified as precachexia, nine as cachexia, and four as refractory cachexia, and the median dose-adjusted fentanyl concentrations ( $\mathrm{ng} / \mathrm{mL}$ per $\mathrm{mg} / \mathrm{kg} /$ day) were $27.5,34.4$, and 44.5, respectively. The dose-adjusted fentanyl concentration in patients with refractory cachexia was higher than that in patients with precachexia (Kruskal-Wallis test and post hoc Mann-Whitney $U$-test, $P<0.01$ ). The factors that were found to possibly affect the dose-adjusted concentration of fentanyl included aspartate aminotransferase, C-reactive protein, and estimated glomerular filtration rate, when analyzed as six independent variables (multiple regression analysis, $P<0.05$ ).

Conclusion: The dose-adjusted plasma concentrations of fentanyl increased with progression of cancer cachexia. Such an increase is associated with a multifactorial and systemic syndrome in cancer cachexia patients, including lower albumin, higher C-reactive protein, and impaired kidney function. In patients with cancer cachexia, we suggest that evaluation of cancer cachexia might help pain management when using a transdermal fentanyl patch in palliative care.

Keywords: transdermal patch, cancer pain, LC-MS/MS

\section{Background}

Fentanyl, a potent opioid, is widely used for cancer pain in step 3 of the World Health Organization (WHO) pain ladder. Unfortunately, it is ineffective for some patients. Pain is prevalent in cancer patients and has been reported in approximately $60 \%$ of patients with either metastatic, advanced stage, or terminal stage disease and in $50 \%$ of cancer patients. ${ }^{1}$ Therefore, good pain control is very important for cancer patients, to maintain their quality of life. Individual differences have been reported in analgesic effects obtained by cancer patients administered fentanyl. ${ }^{2,3}$ Individual variations, in either metabolizing enzymes or skin absorption from the fentanyl transdermal patches, may affect blood fentanyl concentrations. ${ }^{4,5}$ However, information on individual
Department of Oncology Pharmaceutical Care and Sciences, Graduate School of Medicine, Dentistry and Pharmaceutical Sciences Okayama University,

2-5-I, Shikata-cho, Kita-ku,

Okayama 700-8558, Japan

Tel +8I 862356597

Fax +8I 862356597

Email sunoma@pharm.okayama-u.ac.jp 
differences in pain control achieved by fentanyl is limited. A more detailed investigation is needed in order to safely and effectively administer fentanyl.

Cachexia, which occurs in $50 \%-80 \%$ of all cancer patients, has been defined as a multifactorial syndrome that is characterized by progressive disorders of both metabolism and physiological function. ${ }^{6,7}$ The pathophysiology is characterized by a negative protein and energy balance that is driven by both reduced food intake and abnormal metabolism. ${ }^{6-8}$ Cachexia is known to affect metabolizing enzymes, such as CYP3A4 which converts fentanyl to norfentanyl., ${ }^{49}$ A previous report showed that cancer cachexia raises the plasma concentrations of oxycodone, a potent opioid that is metabolized, via CYP3A, as fentanyl. ${ }^{10}$ An understanding of the effect of cachexia on fentanyl plasma concentrations may be beneficial for pain management.

We analyzed fentanyl concentrations in patient plasma. Cancer cachexia was also examined as a factor that may influence plasma fentanyl concentrations. ${ }^{6}$ Cancer cachexia was classified into three stages, using the criteria by Fearon et al. ${ }^{6}$ The aim of this study was to evaluate the influence of cancer cachexia on dose-adjusted plasma fentanyl concentrations in cancer patients.

\section{Methods}

\section{Ethics approval}

This study was designed and implemented following the guidelines dictated in the Declaration of Helsinki. Ethics approval was obtained from the Okayama University Hospital Ethics Committee. Patients in this study remained anonymous and could not be identified. Informed consent was obtained from patients registered in the study.

\section{Patients and plasma samples}

A total of 21 subjects participated in this study between January 2012 and August 2013. Pain control was managed by a palliative care team at Okayama University Hospital. Patient information, including sex, age, height, weight, cancer type, cancer stage, and daily dose of the transdermal fentanyl patch, was obtained from medical records. Exclusion criteria included use of drugs as supportive therapy, that might affect the metabolism of CYP3A4 (eg, erythromycin, diltiazem, itraconazole, and cimetidine), and antipsychotic drugs.

The patients were treated daily with a new 24-hour, transdermal fentanyl patch (Fentos ${ }^{\circledR}$ tape; Hisamitsu Pharmaceutical Co., Saga, Japan, and Kyowa Hakko Kirin Co., Ltd., Tokyo, Japan) to reduce cancer pain. Patients without a change in baseline dose for 3 days were judged to have completed the titration. Blood sampling was performed on days 4, 6, 8, 10, and 14. Blood samples were collected from the patients just before applying a fresh patch. Whole blood was collected in a tube containing heparin, and plasma was separated by centrifugation (3,000 rpm, 15 minutes) and then stored at $-30^{\circ} \mathrm{C}$.

\section{Evaluation of cancer cachexia and laboratory data}

Cancer cachexia was classified as precachexia, cachexia, and refractory cachexia, using diagnostic criteria described by Fearon et al. ${ }^{6}$

Laboratory data were collected to identify the factors that affect fentanyl plasma concentrations. Aspartate aminotransferase (AST) and alanine aminotransferase (ALT) were analyzed to determine liver function. Serum creatinine (S-Cr) and the estimated glomerular filtration rate (eGFR) were analyzed to determine kidney function. In addition, albumin (ALB) and C-reactive protein (CRP) were assessed. Fentanyl concentrations were determined using liquid chromatography (LC) with tandem mass spectrometry (MS/MS) (LC-MS/ MS). Dose-adjusted plasma concentrations of fentanyl were calculated using the following equation:

$$
\begin{aligned}
& \text { Dose-adjusted plasma Plasma concentration of }
\end{aligned}
$$

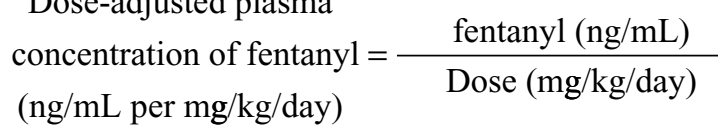

\section{Fentanyl assay}

Fentanyl concentrations were determined using LC-MS/MS. Haloperidol was used as an internal standard. The LC-20AD (Shimadzu Corp, Kyoto, Japan) high-performance LC system and the API 4000TM (AB SCIEX, Framingham, MA, USA) MS/MS system were used. A Capcell Pak C18 AQ type $(2.0 \times 150 \mathrm{~mm}, 5 \mu \mathrm{m}$; Shiseido, Tokyo, Japan) column was used for the analytical column, and the temperature was set to $40^{\circ} \mathrm{C}$. Data were acquired and processed using Analyst ${ }^{\circledR}$ version 1.4.2 (AB SCIEX) software. Assay accuracies at 0.05, 0.5, 1.0 , and $10.0 \mathrm{ng} / \mathrm{mL}$ were $95.2 \%, 99.2 \%, 115.4 \%$, and $98.5 \%$ (intra-assay) and 94.0\%, 99.6\%, 111.7\%, and 98.9\% (interassay), respectively. Assay precisions at $0.05,0.5,1.0$, and 10.0 $\mathrm{ng} / \mathrm{mL}$ were $8.85 \%, 4.37 \%, 4.11 \%$, and $2.64 \%$ (intra-assay) and $8.29 \%, 4.01 \%, 5.64 \%$, and $3.72 \%$ (interassay), respectively. All of these results were within the acceptable range of the US Food and Drug Administration (FDA) guidance. ${ }^{11}$ 


\section{Evaluation of pain intensity and adverse events}

An evaluation of the pain intensity was performed, using a visual analog scale (VAS) (0-100 mm), just before applying a fresh patch. The history of adverse events, which included malaise, somnolence, and constipation, was obtained from the medical records. The severity of adverse events was assessed according to the National Cancer Institute Common Terminology Criteria for Adverse Events (NCI-CTCAE) version $4.0 .^{12}$

\section{Statistical analysis}

All statistical analyses were performed using SPSS (SPSS ${ }^{\circledR}$ Statistics 22.0; IBM Japan, Tokyo, Japan). The Kruskal-Wallis test and the post hoc Mann-Whitney $U$-test with a Bonferroni correction were used to compare the dose-adjusted concentration of fentanyl with the severity of cachexia and to compare each laboratory finding with the stage of cancer cachexia. Multiple regression analysis was performed to identify the factors affecting the dose-adjusted concentration of fentanyl. The dose-adjusted plasma concentration of fentanyl was defined as the dependent variable, and AST, ALT, S-Cr, eGFR, ALB, and CRP were defined as the independent variables. The $\beta$ values indicated dependent variables (variations in doseadjusted plasma fentanyl concentrations) for certain amounts of changes in each of the six independent variables. Data were expressed as the median and interquartile range unless otherwise stated. $P$-values below 0.05 indicated significance.

\section{Results}

\section{Patient demographics and characteristics}

Patient information obtained from the medical records is shown in Table 1. Fifteen types of cancers were identified in 21 patients. The mean VAS score of the patients in this study was 25 (range 10-35) (Table 2). The VAS scores of all patients during the study period were stable. Adverse events were observed in six patients. Malaise and somnolence were observed in three and in four patients, respectively (Table 2).

\section{Influence of cancer cachexia on dose-adjusted fentanyl concentrations}

The dose-adjusted concentration of fentanyl (ng/mL per mg/ $\mathrm{kg}$ /day) (interquartile range) was 27.5 (15.6 -43.7) in patients with precachexia, 34.4 (19.7 -50.7) in those with cachexia, and 44.5 (27.3 -62.8) in those with refractory cachexia (Figure 1). Based on the results of the Kruskal-Wallis test
Table I Patient demographics

\begin{tabular}{ll}
\hline & All patients $(\mathbf{n}=\mathbf{2}$ I) \\
\hline Age in years & $55.3 \pm 11.0$ \\
Height $(\mathrm{cm})$ & $159.1 \pm 10.0$ \\
Weight $(\mathrm{kg})$ & $55.4 \pm 15.1$ \\
Sex: female/male & $14 / 7$ \\
Cancer type & \\
$\quad$ Cervical & 3 \\
Rectal & 2 \\
Endometrial & 2 \\
Ovarian & 2 \\
Adenocarcinoma of lung & 2 \\
Renal & 2 \\
Other & 8 \\
Cancer stage: I/II/III/IV & $1 / 2 / 5 / I 3$ \\
\hline
\end{tabular}

Note: Age in years, Height, and Weight are expressed as mean \pm SD. Sex, Cancer type, and Cancer stage are expressed as number of patients.

Abbreviation: SD, standard deviation.

and post hoc Mann-Whitney $U$-test, with a Bonferroni correction to compare the median dose-adjusted fentanyl concentrations with stages of cachexia, the dose-adjusted fentanyl concentration was significantly higher in patients with refractory cachexia than that in patients with precachexia (Figure 1) $(P<0.005)$.

\section{Patient characteristics based on stages of cancer cachexia}

Body weight was significantly lower in patients with either cachexia or refractory cachexia than in patients with precachexia, and body mass index (BMI) decreased with the progression of cachexia (Table 3 ). ALB was significantly lower in patients with either cachexia or refractory cachexia than in patients with precachexia, and CRP was significantly higher in patients with either cachexia or refractory cachexia than in patients with precachexia. In cachexia patients, variations in both AST and ALT were within the normal range. On the other hand, although variations in S-Cr were within the normal range, eGFR in patients with cachexia and refractory cachexia was significantly lower.

\section{Relationship between the concentration of fentanyl and laboratory data}

Multiple regression analysis, using SPSS, was performed to identify the factors affecting the dose-adjusted concentration of fentanyl. The results obtained are shown in Table 4. Three factors - AST, CRP, and eGFR - were found that might affect the dose-adjusted concentration of fentanyl $(P<0.05)$. On the other hand, there was no significant effect of ALB, ALT, or S-Cr on the dose-adjusted concentration 
Table 2 Characteristics of fentanyl treatment

\begin{tabular}{|c|c|c|c|c|c|}
\hline $\begin{array}{l}\text { Patient } \\
\text { number }\end{array}$ & $\begin{array}{l}\text { Stage of cancer } \\
\text { cachexia }\end{array}$ & $\begin{array}{l}\text { Dose of fentanyl } \\
\text { (mg/kg/day) }\end{array}$ & $\begin{array}{l}\text { Dose-adjusted plasma concentration } \\
\text { of fentanyl ( } \mathrm{ng} / \mathrm{mL} \text { per } \mathrm{mg} / \mathrm{kg} / \text { day })\end{array}$ & $\begin{array}{l}\text { VAS score } \\
(\mathrm{mm})\end{array}$ & Adverse events \\
\hline 1 & PC & 0.097 & 29.9 & 25 & \\
\hline 2 & PC & 0.043 & 15.2 & 26 & \\
\hline 3 & PC & 0.091 & 15.6 & 30 & \\
\hline 4 & $\mathrm{PC}$ & 0.049 & 19.8 & 10 & \\
\hline 5 & PC & 0.018 & 31.4 & 22 & Constipation \\
\hline 6 & PC & 0.080 & 45.9 & 27 & \\
\hline 7 & PC & 0.058 & 30.1 & 16 & \\
\hline 8 & C & 0.215 & 27.5 & 19 & \\
\hline 9 & C & 0.010 & 43.7 & 20 & \\
\hline 10 & $\mathrm{C}$ & 0.020 & 15.5 & 25 & \\
\hline 11 & C & 0.037 & 50.1 & 19 & \\
\hline 12 & C & 0.168 & 57.3 & 20 & Constipation \\
\hline 13 & C & 0.056 & 37.2 & 26 & \\
\hline 14 & C & 0.074 & 16.7 & 28 & \\
\hline 15 & $\mathrm{C}$ & 0.230 & 54.8 & 33 & Malaise \\
\hline 16 & C & 0.117 & 32.1 & 22 & \\
\hline 17 & C & 0.045 & 36.3 & 26 & \\
\hline 18 & $\mathrm{RC}$ & 0.282 & 52.1 & 30 & Somnolence, malaise \\
\hline 19 & $\mathrm{RC}$ & 0.199 & 18.5 & 29 & \\
\hline 20 & $\mathrm{RC}$ & 0.045 & 44.6 & 35 & Somnolence, malaise \\
\hline 21 & $\mathrm{RC}$ & 0.021 & 55.0 & 32 & Somnolence, malaise \\
\hline
\end{tabular}

Note: VAS scores represent the mean value.

Abbreviations: C, cachexia; PC, precachexia; RC, refractory cachexia; VAS, visual analog scale.

of fentanyl. Positive $\beta$ indicated that the factor influenced the dependent variable (variations in dose-adjusted fentanyl concentrations) in a positive direction. Accordingly, the $\beta$ was positive for AST, and an increase in the AST value

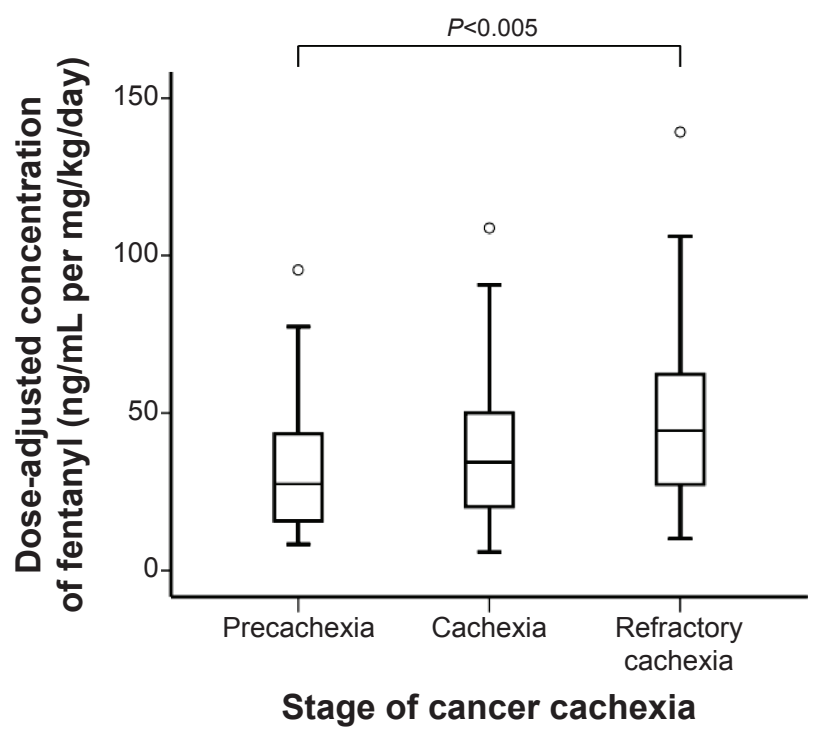

Figure I The relationship between stage of cancer cachexia and the dose-adjusted plasma concentration of fentanyl.

Notes: Statistical analysis was performed using the Kruskal-Wallis test and the post hoc Mann-Whitney U-test, with a Bonferroni correction. The $P$-values were from the Mann-Whitney $U$-test. Box plots represent the median, 25th, and 75th percentiles. The range within 1.5 times the length of the interquartile is shown. Outliers (values that were between 1.5 and 3 times the interquartile range) are represented by an open circle. may elevate the dose-adjusted concentration of fentanyl ( $\beta=0.198, P=0.010$ ). Similarly, the $\beta$ was positive for CRP, and the adjusted concentration of fentanyl may increase as CRP increases ( $\beta=0.216, P=0.003$ ). On the other hand, the $\beta$ was negative for eGFR, and the dose-adjusted concentration of fentanyl may increase as the eGFR decreases $(\beta=-0.571$, $P<0.001)$.

\section{Discussion}

Our study showed that the dose-adjusted concentration of fentanyl was increased in patients with either cachexia or refractory cachexia. We demonstrated that such an increase was associated with a multifactorial and systemic syndrome in cancer cachexia patients, including lower ALB, higher $\mathrm{CRP}$, and impaired kidney function. We presume that metabolic abnormalities in cachexia, such as inhibition of CYP3A4 and decline of renal function, might cause higher plasma concentrations of fentanyl. Therefore, evaluation of cancer cachexia might help pain management when using the transdermal fentanyl patch in palliative care.

Cancer cachexia is characterized by the ongoing loss of skeletal muscle mass, with or without the loss of fat mass; lack of a full response to conventional nutritional support; and progressive metabolic and physiological functional impairments. ${ }^{6,7}$ The pathophysiology of cachexia is characterized by a negative protein and energy balance 
Table 3 Patient characteristics relative to stage of cancer cachexia

\begin{tabular}{|c|c|c|c|c|}
\hline \multirow[t]{2}{*}{ Characteristics } & \multirow[t]{2}{*}{ All patients $(n=2 I)$} & \multicolumn{3}{|c|}{ Stage of cancer cachexia } \\
\hline & & Precachexia $(n=8)$ & Cachexia $(n=9)$ & Refractory cachexia $(n=4)$ \\
\hline Sex: female/male & $14 / 7$ & $5 / 3$ & $7 / 2$ & $2 / 2$ \\
\hline Age (years) & $56(49-63)$ & $50(48-52)$ & $62(57-67)^{*}$ & $57(49-7 I)$ \\
\hline Height (cm) & $156(152-165)$ & $162(155-169)$ & $154(149-161)$ & $158(|52-17|)$ \\
\hline Weight (kg) & $54.3(44.5-65.0)$ & $69.8(61.0-73.9)$ & $52.0(43.5-54.3)^{* *}$ & $48.3(38.2-63.9)^{* *}$ \\
\hline BMI $\left(\mathrm{kg} / \mathrm{m}^{2}\right)$ & $20.0(18.6-22.5)$ & $23.1(22.8-32.4)$ & $19.0(18.3-19.8)^{* *}$ & $19.8(18.0-20.9)^{* *}$ \\
\hline Dose $(\mathrm{mg} / \mathrm{kg} /$ day) & $0.097(0.049-0.207)$ & $0.080(0.049-0.105)$ & $0.099(0.056-0.282)^{* *}$ & $0.156(0.044-0.219)$ \\
\hline Fentanyl plasma concentration $(\mathrm{ng} / \mathrm{mL})^{\#}$ & $3.20(1.40-5.69)$ & $1.95(1.00-3.26)$ & $3.90(1.62-9.33)^{* *}$ & $3.73(2.20-7.56)^{* *}$ \\
\hline ALB (g/L) & $32.0(25.0-37.0)$ & $36.0(31.0-39.0)$ & $32.0(21.0-37.0)^{* *}$ & $25.0(23.0-29.0)^{* *}$ \\
\hline AST (IU/L) & $22.0(16.0-36.5)$ & $37.0(19.0-48.0)$ & $20.0(17.0-26.0)^{* *}$ & $20.0(12.0-33.8)^{* *}$ \\
\hline ALT (IU/L) & $16.0(10.0-23.0)$ & $19.0(12.0-33.0)$ & $14.0(10.0-19.0)^{* *}$ & $11.5(6.8-22.5)^{* *}$ \\
\hline $\mathrm{S}-\mathrm{Cr}(\mathrm{mg} / \mathrm{dL})$ & $0.62(0.50-0.87)$ & $0.59(0.44-0.66)$ & $0.60(0.50-1.03)$ & $0.74(0.64-1.06)^{* *}$ \\
\hline eGFR (mL/min) & $89.3(60.2-122.4)$ & I I 3.8 (87.7-|43.4) & $77.8(53.9-121.6)^{* *}$ & $66.8(42.9-101.2)^{* *}$ \\
\hline CRP (mg/L) & $24.0(6.7-61.5)$ & $6.6(3.5-32.0)$ & $17.5(7.8-5 \mid .2)^{*}$ & $70.0(39.2-103.0)^{* *}$ \\
\hline
\end{tabular}

Notes: Data are expressed as the median (interquartile range). $* P<0.05$, $* * P<0.0$ I, significantly different from patients classified with precachexia. ${ }^{*}$ Measured value of fentanyl plasma concentration.

Abbreviations: ALB, albumin; ALT, alanine aminotransferase; AST, aspartate aminotransferase; BMI, body mass index; CRP, C-reactive protein; eGFR, estimated glomerular filtration rate; $\mathrm{S}-\mathrm{Cr}$, serum creatinine.

driven by the variable combination of reduced food intake and abnormal metabolism. ${ }^{6,7}$ Cachexia is known to affect metabolizing enzymes, such as CYP3A4, which converts fentanyl to norfentanyl., 4

In this study, dose-adjusted plasma concentrations of fentanyl increased with the progression of cancer cachexia. Although the influence of cancer cachexia on the plasma concentrations of fentanyl has been previously reported, the previously published results differ from our findings. The previous study reported that the plasma concentrations of fentanyl were lower in cachectic patients because absorption from the transdermal fentanyl patch was impaired in cachectic patients. ${ }^{13} \mathrm{We}$ collected blood samples during the steady state of fentanyl plasma concentration, while blood sampling in the previous study was performed for 3 days after application of the first patch, before steady state was

Table 4 Predictive factors for the dose-adjusted plasma concentration of fentanyl

\begin{tabular}{lllll}
\hline Factors & $\mathbf{B}$ & Std error & $\beta$ & $t(p)$ \\
\hline Constant & 1.682 & 0.147 & & $I I .4 I(<0.00 \mathrm{I})$ \\
eGFR & -0.010 & $0.00 \mathrm{I}$ & $-0.57 \mathrm{I}$ & $-7.430(<0.00 \mathrm{I})$ \\
CRP & 0.004 & $0.00 \mathrm{I}$ & 0.216 & $3.068(0.003)$ \\
AST & 0.008 & 0.003 & 0.198 & $2.615(0.010)$ \\
ALB & & & -0.077 & $-0.793(0.429)$ \\
ALT & & & -0.140 & $-1.850(0.067)$ \\
S-Cr & & & -0.148 & $-1.527(0.129)$ \\
\hline
\end{tabular}

Notes: The $F$ score of this model was significant $(F=25.17, P<0.00 I)$, with adjusted $R^{2}=0.348$. B signifies unstandardized coefficients; $\beta$ signifies standardized coefficients. Abbreviations: ALB, albumin; ALT, alanine aminotransferase; AST, aspartate aminotransferase; CRP, C-reactive protein; eGFR, estimated glomerular filtration rate; $\mathrm{S}-\mathrm{Cr}$, serum creatinine; Std error, standard error. achieved. In addition, we used a 24-hour transdermal fentanyl patch, while the previous study used a 72-hour transdermal fentanyl patch. We believe that these differences in methodology explain the difference between our findings and those of the previous report.

Our results, obtained by multiple regression analysis, indicated that a reduction in the eGFR may increase fentanyl plasma concentrations. Approximately $90 \%$ of fentanyl is converted to norfentanyl in the liver by CYP3A4, and the other $10 \%$ is excreted unchanged by the kidney. ${ }^{4}$ Norfentanyl, the main metabolite of fentanyl, is inactive and has few effects as a stored metabolite; therefore, fentanyl can generally be administered to patients with kidney failure. However it was reported that clearance of fentanyl in patients with kidney failure was lower than that in patients without kidney failure. ${ }^{14}$ Although fentanyl is recommended for severe pain for patients with chronic kidney disease, reduction in dose and frequency may be advisable for these patients. ${ }^{15}$ Our results also suggested that the eGFR should be evaluated when administering fentanyl to kidney failure patients. Therefore, we suggest that a lower kidney function in patients with cancer cachexia might result in an increase in the plasma concentration of fentanyl.

Our results also indicated that an increase in both CRP and AST may elevate the plasma concentration of fentanyl. Cachexia is known to influence drug metabolism, which may affect fentanyl levels. ${ }^{9}$ Mantovani et al previously reported that the inflammatory cytokines, which induce the production of acute-phase proteins, including CRP, and inhibit the synthesis of ALB in the liver, were elevated with the progression of 
cachexia. ${ }^{16}$ High CRP and low ALB may be caused by the increased release of inflammatory cytokines in cancer cachexia patients. It was reported that inflammatory cytokines caused a reduction in the levels of CYP3A4 messenger (m)RNA and downregulated the expression of CYP3A4. ${ }^{17,18}$ Naito et al reported that cachexia raised the plasma concentrations of oxycodone, a potent opioid that is metabolized, via CYP3A4, as fentanyl. ${ }^{10}$ They explained that the plasma concentrations of oxycodone in cachexia patients might be increased by a reduction in CYP3A activity. We suggest that the systemic inflammation occurring with the progression of cachexia might decrease the metabolism of fentanyl by CYP3A4 in the liver and thereby increase the concentration of fentanyl in the blood. Although inflammatory cytokines may influence liver function, the relationship between inflammation and AST has yet to be elucidated in detail.

Although our sample size was limited, we demonstrated that the higher plasma concentration of fentanyl in patients with either cachexia or refractory cachexia was associated with a multifactorial and systemic syndrome, including lower ALB, higher CRP, and impaired kidney function. Therefore, the severity of cachexia should be considered when determining the dose of fentanyl, in order to prevent adverse events caused by too high plasma concentrations of fentanyl. The influence of cancer cachexia on the plasma concentrations of fentanyl should be evaluated in larger trials.

The important point in transdermal fentanyl therapy is that there is significant interpatient variability in plasma drug concentration. One of our patients with refractory cachexia received a fentanyl patch at a dose of $18 \mathrm{mg} /$ day and suffered somnolence (grade 3 ). The patient's plasma fentanyl concentration was $14.7 \mathrm{ng} / \mathrm{mL}$. The dose of fentanyl was then reduced to $10 \mathrm{mg} / \mathrm{day}$, and the plasma concentration of fentanyl decreased to $5.8 \mathrm{ng} / \mathrm{mL}$, resulting in good pain control without somnolence. An optimal therapeutic concentration of fentanyl has not yet been established. The interview form for the transdermal fentanyl patch provided by the company contained the following information: ${ }^{19}$ Although the effective plasma concentration of fentanyl in patients with no opioid resistance is generally $0.2-1.2 \mathrm{ng} / \mathrm{mL}$, when either pain increases or pain tolerance occurs, the effective plasma concentration also increases. In the present case, reduction in the dose of fentanyl was needed due to severe somnolence, but the plasma concentration of fentanyl in this case was higher than that range. This case indicates that evaluation of cachexia could lead to good pain control using the fentanyl patch, in hard-to-manage patients.

\section{Conclusion}

The dose-adjusted plasma concentrations of fentanyl increased with progression of cancer cachexia. Such an increase is associated with a multifactorial and systemic syndrome in cancer cachexia patients, including lower ALB, higher CRP, and impaired kidney function. In patients with cachexia, we suggest that evaluation of cachexia might help pain management using a transdermal fentanyl patch in palliative care.

\section{Disclosure}

The authors report no conflicts of interest in this work.

\section{References}

1. van den Beuken-van Everdingen MH, de Rijke JM, Kessels AG, Schouten HC, van Kleef M, Patijn J. Prevalence of pain in patients with cancer: a systematic review of the past 40 years. Ann Oncol. 2007;18(9): 1437-1449.

2. Menahem S, Shvartzman P. High-dose fentanyl patch for cancer pain. J Am Board Fam Pract. 2004;17(5):388-390.

3. Radbruch L, Sabatowski R, Petzke F, Brunsch-Radbruch A, Grond S, Lehmann KA. Transdermal fentanyl for the management of cancer pain: a survey of 1005 patients. Palliat Med. 2001;15(4):309-321.

4. Labroo RB, Paine MF, Thummel KE, Kharasch ED. Fentanyl metabolism by human hepatic and intestinal cytochrome P450 3A4: implications for interindividual variability in disposition, efficacy, and drug interactions. Drug Metab Dispos. 1997;25(9):1072-1080.

5. Grond S, Radbruch L, Lehmann KA. Clinical pharmacokinetics of transdermal opioids: focus on transdermal fentanyl. Clin Pharmacokinet. 2000;38(1):59-89.

6. Fearon K, Strasser F, Anker SD, et al. Definition and classification of cancer cachexia: an international consensus. Lancet Oncol. 2011; 12(5):489-495.

7. Radbruch L, Elsner F, Trottenberg P, Strasser F, Fearon K. Clinical Practice Guidelines on Cancer Cachexia in Advanced Cancer Patients with a Focus on refractory Cachexia. Aachen: Department of Palliative Medicine, European Palliative Care Research Collaborative; 2010. Available from: http://www.epcrc.org/guidelines.php? $\mathrm{p}=$ cachexia. Accessed February 15, 2015.

8. Douglas E, McMillan DC. Towards a simple objective framework for the investigation and treatment of cancer cachexia: the Glasgow Prognostic Score. Cancer Treat Rev. 2014;40(6):685-691.

9. Slaviero KA, Clarke SJ, Rivory LP. Inflammatory response: an unrecognised source of variability in the pharmacokinetics and pharmacodynamics of cancer chemotherapy. Lancet Oncol. 2003;4(4):224-232.

10. Naito T, Tashiro M, Ishida T, Ohnishi K, Kawakami J. Cancer cachexia raises the plasma concentration of oxymorphone through the reduction of CYP3A but not CYP2D6 in oxycodone-treated patients. $J$ Clin Pharmacol. 2013;53(8):812-818.

11. Food and Drug Administration [homepage on the Internet]. Silver Spring: Guidance for industry analytical procedures and methods validation for drugs and biologics. [updated $2014 \mathrm{Feb}$; cited 2014 Sep 10] Available from: http://www.fda.gov/downloads/Drugs/GuidanceComplianceRegulatoryInformation/Guidances/UCM386366.pdf. Accessed September 10, 2014.

12. National Institutes of Health [homepage on the Internet]. Bethesda: Common Terminology Criteria for Adverse Events (CTCAE) Version 4.03, vol. 2009; 2010. [updated 2010 Jun 14; cited 2014 Sep 10] Available from: http://evs.nci.nih.gov/ftp1/CTCAE/CTCAE_4.03_2010-06-14_ QuickReference_8.5x11.pdf. Accessed September 10, 2014. 
13. Heiskanen T, Mätzke S, Haakana S, Gergov M, Vuori E, Kalso E. Transdermal fentanyl in cachectic cancer patients. Pain. 2009;144 (1-2):218-222.

14. Koehntop DE, Rodman JH. Fentanyl pharmacokinetics in patients undergoing renal transplantation. Pharmacotherapy. 1997;17(4): 746-752.

15. Pham PCT, Toscano E, Pham PMT, Pham PAT, Pham SV, Pham PTT. Pain management in patients with chronic kidney disease. NDT Plus. 2009; 2(2):111-118.

16. Mantovani G, Macciò A, Madeddu C, et al. Quantitative evaluation of oxidative stress, chronic inflammatory indices and leptin in cancer patients: correlation with stage and performance status. Int J Cancer. 2002; 98(1):84-91.
17. Rivory LP, Slaviero KA, Clarke SJ. Hepatic cytochrome P450 3A drug metabolism is reduced in cancer patients who have an acute-phase response. Br J Cancer. 2002;87(3):277-280.

18. Jover R, Bort R, Gómez-Lechón MJ, Castell JV. Down-regulation of human CYP3A4 by the inflammatory signal interleukin-6: molecular mechanism and transcription factors involved. FASEB J. 2002;16(13):1799-1801.

19. Fentos ${ }^{\circledR}$ tape [Drug interview form]. Saga, Japan: Hisamitsu Pharmaceutical Co., Inc. and Tokyo: Kyowa Hakko Kirin Co., Ltd.; 2011.

Therapeutics and Clinical Risk Management

\section{Publish your work in this journal}

Therapeutics and Clinical Risk Management is an international, peerreviewed journal of clinical therapeutics and risk management, focusing on concise rapid reporting of clinical studies in all therapeutic areas, outcomes, safety, and programs for the effective, safe, and sustained use of medicines. This journal is indexed on PubMed Central, CAS,
EMBase, Scopus and the Elsevier Bibliographic databases. The manuscript management system is completely online and includes a very quick and fair peer-review system, which is all easy to use. Visit http://www.dovepress.com/testimonials.php to read real quotes from published authors.

Submit your manuscript here: http://www.dovepress.com/therapeutics-and-clinical-risk-management-journal 